\title{
Effects of local structure on helium bubble growth in bulk and at grain boundaries of bec iron: a molecular dynamics study
}

\author{
L. Yang, ${ }^{\mathrm{a},{ }^{*}}$ F. Gao, ${ }^{\mathrm{c},}{ }^{\dagger}$ R.J. Kurtz, ${ }^{\mathrm{b}}$ X. T. Zu, ${ }^{\mathrm{a}}$ S.M. Peng, ${ }^{\mathrm{d}}$ X.G. Long, ${ }^{\mathrm{d}}$ X.S. Zhou ${ }^{\mathrm{d}}$ \\ ${ }^{a}$ School of Physical Electronics, University of Electronic Science and Technology of China, \\ Chengdu 610054, China \\ ${ }^{\mathrm{b}}$ Pacific Northwest National Laboratory, Richland, WA 99352, USA \\ ${ }^{\mathrm{c}}$ Department of Nuclear Engineering and Radiological Sciences, University of Michigan, Ann \\ Arbor, Michigan 48109, USA \\ ${ }^{\mathrm{d}}$ Institute of Nuclear Physics and Chemistry, China Academy of Engineering Physics, \\ Mianyang 621900, China
}

\footnotetext{
* Corresponding author. Tel: (+86) 028-8320-2130; Fax: (+86) 028-8320-2130; e-mail: yanglildk@uestc.edu.cn

${ }^{\dagger}$ Corresponding author. Tel: (+1) 509-371-6490; Fax: (+1) 509-371-6242; e-mail: fei.gao@pnnl.gov
} 


\begin{abstract}
The nucleation and growth of helium (He) bubbles in the bulk and at $\Sigma 3<110>\{112\}$ and $\sum 73 \mathrm{~b}<110>\{661\}$ grain boundaries (GBs) in bcc iron have been investigated using molecular dynamics simulations. The results show that a $1 / 2<111>\{111\}$ dislocation loop is formed with the sequential collection of $<111>$ interstitial crowdions at the periphery of the He cluster and is eventually emitted from the He cluster. Insertion of $45 \mathrm{He}$ atoms into a He cluster leads to the formation of a $1 / 2<111>$ dislocation loop in $\Sigma 3 \mathrm{~GB}$. It is of interest to notice that the transition of a dislocation segment through the GB leads to the formation of a step at the GB plane following the loop formation, accounting for the formation of a residual GB defect. A $1 / 2<111>$ loop, with a $\{110\}$ habit plane, is emitted with further increase of the He bubble size in the $\Sigma 3 \mathrm{~GB}$. In contrast, the sequential insertion of He atoms in $\sum 73 \mathrm{~b}$ GB continuously emits selfinterstitial atoms (SIAs), but these SIAs rearrange at the core of the inherent GB dislocation, instead of forming a dislocation loop, which leads the GB dislocation to propagate along the $[\overline{1} \overline{1} 12]$ direction. In the bulk and $\Sigma 3 \mathrm{~GB}$, the He bubble exhibits three-dimensionally spherical shape, but it forms longitudinal shape along the dislocation line in the $\sum 73 \mathrm{~GB}$, a shape commonly observed at GBs in experiments.
\end{abstract}

Keywords: Grain boundaries; Atomistic simulation; He bubble growth; Bcc iron; Dislocation loops 


\section{INTRODUCTION}

It is well known that grain boundaries (GBs) and interfaces are effective sinks for radiation-induced defects [1-3]. Bai and co-workers have investigated defect-GB interaction mechanisms in copper and shown that GBs capture interstitials and inject them back into the lattice to recombine with vacancies that are within a few nanometers of the GB, leaving a healed crystal [4]. Because of the extremely low solubility of helium $(\mathrm{He})$ in metallic materials, high concentrations of He created by transmutation are known to induce the formation of He bubbles at pre-existing and radiation-induced defects, and significantly degrade the mechanical properties of first wall structural materials [2,5-7]. The formation of He bubbles in the bulk or at GBs remains one of the most important issues in nuclear fusion technology, and He accumulation, both in the bulk and at GBs, has major consequences for the structural integrity of nuclear materials. Sefta et al. have investigated the tungsten surface evolution due to helium bubble nucleation, growth and rupture using molecular dynamics (MD) simulations [8]. MD simulations of helium implantation into single-crystalline tungsten were performed and it was found that the clusters were nucleated via the creation of (111) crowdion interstitials and interstitial dislocation loop punching [9]. The growth of helium clusters in titanium crystals was simulated using MD, and defect escape from the helium cluster was observed at certain points in the growth process [10]. At the microscopic level, understanding the clustering mechanism of $\mathrm{He}$ atoms, the formation of He-vacancy $(\mathrm{He}-\mathrm{V})$ clusters and the growth of a He cluster into a bubble is a prerequisite for simulating more complex phenomena at longer time scales and larger He concentrations. Zhang et al. investigated the energetic landscape and diffusion of $\mathrm{He}$ at grain boundaries in $\alpha$-Fe from first principles and found the diffusion details and precise paths are GB dependent [11].

The nucleation of He clusters and bubbles in the bulk and at the GBs in $\alpha$-Fe has been previously studied to understand the formation and evolution of these defects, and the effects of these phenomena on microstructural changes [12-16]. These 
investigations showed that the evolution of GB structure, the accumulation of He atoms and the nucleation of $\mathrm{He}$ bubbles all depend on the He concentration, irradiation temperature and the original GB structure. In our previous work we found that $<100>$ dislocation loops can directly form in the region between large He clusters in the $\Sigma 3 \mathrm{~GB}$ at 600 and $800 \mathrm{~K}^{8}{ }^{8}$ At high He concentrations, a large number of self-interstitial atoms (SIAs) are emitted from He clusters and these SIAs are able to rearrange to form an extra atomic plane within the $\Sigma 3 \mathrm{~GB}$, resulting in the self-healing of the deformation induced by $\mathrm{He}$ accumulation and GB migration. In contrast, the large number of SIAs emitted by He clusters in a $\sum 73$ b GB causes climb of intrinsic GB dislocations parallel to the GB plane, and this climb becomes more significant with increasing temperature. In bulk Fe, at low He concentrations, SIA clusters (or dislocation loops formed from SIA clusters) are attached to $\mathrm{He}-\mathrm{V}$ clusters, while at high $\mathrm{He}$ concentrations $\mathrm{He}-\mathrm{V}$ cluster-loop complexes with more than one He-V cluster are formed. In these studies $[13,14]$. He atoms were randomly positioned at tetrahedral sites in the bulk or the stressed region near the GB plane of $\alpha$-Fe, mimicking the He accumulation at different concentrations. In the present work, $\mathrm{He}$ atoms are inserted one by one to study the growth of a He cluster and the formation and evolution of SIAs in the bulk and at two GBs ( $\sum 3$ and $\left.\sum 73 b\right)$ in $\alpha-F e$, thus providing information on the detailed mechanisms controlling nucleation and growth of He bubbles. It should be noted that Frenkel pairs are also produced within fusion reactor conditions. There are two possible mechanisms that give rise the nucleation of He bubbles, i.e. vacancy-enhanced nucleation of $\mathrm{He}$ bubbles and one due to He accumulation in the bulk and at GBs. The present study focuses on the later case to understand the clustering mechanism of $\mathrm{He}$ atoms and the nucleation of He-vacancy (He-V) clusters

\section{SIMULATION PROCEDURE}


The potentials used in this work are the same as those used in Ref. 8. The Fe-Fe, Fe$\mathrm{He}$ and $\mathrm{He}-\mathrm{He}$ interactions are described by the interatomic potentials of Ackland et al [17], Gao et al [18] and Aziz [19], respectively. This set of potentials provides a good description of bulk properties and defect properties of the Fe-He system, which are in agreement with experimental data and ab inito calculations. The $<110>\mathrm{Fe}-\mathrm{Fe}$ dumbbell configuration is the most stable interstitial. Dynamically, the $<110>$ interstitial may change to the $<111>$ direction, followed by faster one-dimensional migration. The Fe$\mathrm{He}$ potential gives the binding energies of small $\mathrm{He}-\mathrm{V}$ and $\mathrm{He}-\mathrm{He}$ clusters in good agreement with those obtained by $a b$ initio and other potential calculations.

An MD box of $30 a_{0} \times 30 a_{0} \times 30 a_{0}$ with $54,000 \mathrm{Fe}$ atoms is used to simulate the formation and growth of He clusters in bulk iron, where $a_{0}$ is the lattice constant of a perfect bcc iron $(2.8553 \AA)$. Periodic boundary conditions are applied along all three directions. Two symmetric tilt GBs with a common $<101>$ tilt axis are constructed to investigate the formation and growth of He clusters in GBs that exhibit markedly different structure and energy. They are the $\Sigma 3\{112\} \Theta=70.53^{\circ}$ and the $\Sigma 73\{661\} \Theta$ $=13.44^{\circ}$, where the minimum energy atomic structures are described in Ref. 8 . The block sizes of the $\Sigma 3$ and $\Sigma 73$ b GBs are $84.070 \AA \times 79.00 \AA \times 80.69 \AA$ with $45,560 \mathrm{Fe}$ atoms and $103.50 \AA \times 70.00 \AA \times 80.76 \AA$ with $48,000 \mathrm{Fe}$ atoms, respectively. For the two GBs, periodic boundary conditions are imposed along the directions parallel to the GB plane, but fixed boundary conditions are applied normal to the GB plane.

The NVT (constant number of atoms, volume and temperature) ensemble is applied in the present simulations with a time step of 1 fs. Helium atoms are continuously inserted one by one into the same region of the simulation box. After each He atom insertion, the configuration is quenched to $0 \mathrm{~K}$, followed by temperature rescaling to $300 \mathrm{~K}$ and annealing for an additional $100 \mathrm{ps}$ at that temperature. A similar procedure is repeated during the simulations. A similar approach was employed to study SIA emission by a He cluster [20], but with a much fast rate of introducing He atoms. We have carefully tested the rate of introducing He atoms, and found that the rate has small effects on the number of He atoms in a cluster that emits a self-interstitial atom (SIA). 
The nucleation and growth of He bubbles in the present simulations are closely associated with emission of SIAs, and thus the rate would not have significant effects on the results presented in this study. Interstitials and vacancies are identified and counted using a displacement analysis method [21]. If an Fe atom is displaced from its lattice site by at least $0.3 \mathrm{a}_{0}$, this atom is considered to be an SIA; if there is no atom within $0.3 \mathrm{a}_{0}$ of a lattice site, the site is taken to be a vacancy.

\section{RESULTS AND DISCUSSION}

\section{A. He cluster evolution in bulk iron}

The growth of He clusters (or bubbles) in bulk Fe is investigated first, and the results are compared with those at GBs. Recent studies show that single He interstitials and small $\mathrm{He}$ clusters $\left(\mathrm{He}_{2}, \mathrm{He}_{3}\right)$ quickly diffuse through lattice at $300 \mathrm{~K}$, ${ }^{7}$ and thus, the simulation is initialized with four He atoms $\left(\mathrm{He}_{4}\right)$ located at a tetrahedral interstitial site in the centre of the simulation box. The $\mathrm{He}_{4}$ cluster is also able to migrate away from the initial site without creating a SIA. Upon adding the fifth $\mathrm{He}$, the $\mathrm{He}_{5}$ cluster pushes the nearest $\mathrm{Fe}$ atom off its lattice site along the $<111>$ direction creating more space within the He cluster, but the emission of a SIA does not occur. After gradually inserting two more He atoms, the displacements of the Fe atoms around the He cluster increase, as shown in Fig. 1(a). However, Fig. 1(b) clearly shows that an Fe atom is kicked out from the cluster when the number of He atoms increases to eight, forming a $\mathrm{He}_{8} \mathrm{~V}$ cluster and an SIA. The SIA appears as a $<111>$ crowdion with a distance of about $3.5 \AA$ from the centre of the He cluster, but the $<111>$ crowdion can also transform to the $\langle 110\rangle$ dumbbell during annealing. We observed that the minimum number of He atoms in the cluster required to create an SIA is slightly different from that of Refs. [20-23] at $300 \mathrm{~K}$. With different potentials, Morishita et al [22] found that the pressure of a six He atom cluster was large enough to push an Fe atom off from its normal site and create a Frenkel pair. Gao et al. also predicted that a $\mathrm{He}_{6}$ cluster would create an SIA with $<111>$ crowdion configuration [20], but Guo et al. observed that 
three He atoms were sufficient to create an SIA in their simulations [23]. Our result is close to the prediction of Ref. [24], in which a Frenkel pair can be produced by a cluster containing ten $\mathrm{He}$ atoms at $200 \mathrm{~K}$, and nine He atoms at $400 \mathrm{~K}$. The insertion of the eleventh He atom followed by annealing at $300 \mathrm{~K}$ causes a second SIA to be emitted with a $<110>$ dumbbell configuration, as shown in Fig. 1(c). These SIAs collect at the periphery of the He-V cluster. Fig. 1(d) illustrates that after insertion of $13 \mathrm{He}$ atoms, all the SIAs move to the same side of the cluster, rather than remaining uniformly distributed over the cluster surface, which is consistent with Morishita's observations in $\mathrm{Fe}$ [22] and Wilson's results in $\mathrm{Ni}$ [25]. A fourth SIA is emitted from a $15 \mathrm{He}$ atom cluster and then the four SIAs spontaneously transform into [1 11] crowdions during annealing.

Insertion of additional $\mathrm{He}$ atoms results in the accumulation of more SIA atoms at the periphery of the cluster. Several snapshots of the configurations produced by continuously adding $\mathrm{He}$ atoms are shown in Fig. 2, where all the insets are viewed normal to the $<111>$ direction. When the $23^{\text {rd }}$ He atom is introduced, eight SIAs align into the same $<111>$ direction as crowdions, forming a well-defined SIA cluster attached to the He-V cluster, as shown in Fig. 2(a), where the inset is the configuration of the SIAs, vacancies and He atoms projected on the $\{111\}$ plane. A $1 / 2<111>$ dislocation loop with $N_{\mathrm{I}}=16$ (the number of SIAs) is formed after the insertion of 35 He atoms. The inset in Fig. 2(b) shows the 16 SIA cluster configuration on the $\{111\}$ plane, which arrange to form part of a hexagonal dislocation loop. The open circles represent empty positions in this loop. It should be noted that the dislocation loop is still attached to the He cluster. After the $41^{\text {st }} \mathrm{He}$ atom is inserted into the cluster, a dislocation loop with $N_{\mathrm{I}}=18$ is able to move away from the He cluster along a $<111>$ direction. The configurations of the He cluster and nearby defects following 10 ps of annealing are shown in Fig. 2(c). The configurations of interstitial dislocation loops formed under irradiation in Fe-based bcc materials have been investigated in many experimental and atomistic simulation studies [26-35]. In the present work, a 1/2<111> loop is formed during the growth of a He cluster, as shown in Fig. 3. It is obvious that 
this loop is a set of parallel $<111>$ crowdions with their centres in a $\{111\}$ plane. The loop gliding cylinder and extra planes are indicated in Fig. 3 (b), which reveals that the loop has a (111) habit plane.

In order to understand the effect of He bubble pressure on the emission of SIAs and bubble growth, the pressure before and after annealing is calculated using the following equation $[23,36]$ :

$$
P=\frac{1}{3 V} \sum_{i}^{n} \Omega_{i}\left[\sigma_{11}(i)+\sigma_{22}(i)+\sigma_{33}(i)\right]
$$

where $n$ is the number of He atoms in the cluster or bubble, $\sigma_{\alpha \beta}$ is the local stress at the $i$ th He atom site, and $V$ is the volume of the cluster or bubble, which is determined from the Voronoi cell for each He atom using Voro++ [37] software. In Fig. 4, one can see that the pressure in the He cluster almost always decreases after annealing. The overall trend is decreasing bubble pressure with increasing numbers of He atoms. The sharp pressure drops are associated with SIA emission and dislocation loop formation. As noted above adding vacancies through SIA emission increases the effective volume occupied by a cluster.

In the present simulations, a bubble containing $41 \mathrm{He}$ atoms is able to emit a dislocation loop consisting of 18 SIAs, which is smaller than the critical bubble size reported in Ref.15. A larger simulation box $\left(50 a_{0} \times 50 a_{0} \times 50 a_{0}\right)$ with $250,000 \mathrm{Fe}$ atoms was used to check the influence of the box size on the results. The detailed configurations around the He clusters were somewhat different from those in the smaller box $\left(30 a_{0} \times 30 a_{0} \times 30 a_{0}\right)$, but the SIA emission and dislocation loop formation processes were very close. We conclude that the results for both box sizes are essentially the same, differing only in minor details.

\section{B. He cluster evolution in a $\Sigma 3 \mathrm{~GB}$}

Experimental observations and atomistic calculations indicate that GBs act as sinks for trapping He atoms in steels or iron [5, 38-42]. The rate of nucleation and growth of 
He bubbles at GBs are likely to determine the extent of embrittlement and may potentially be one of the most serious material problems related to the development of fusion power. Therefore, the growth of small He bubbles at GBs in bcc iron is investigated with the same approach used in the bulk by inserting He atoms one by one at $300 \mathrm{~K}$. The first He atom is inserted into a tetrahedral site, one of the most favorable interstitial sites in the GB. Once the He cluster reaches size 4, the cluster moves slightly away from the GB plane and a $<111>$ SIA crowdion is created within the GB. The emitted SIA is able to easily migrate along the $<111>$ direction within the GB plane. After the $9^{\text {th }} \mathrm{He}$ is inserted, the displacements of the Fe atoms around the He cluster increase, leading to a few Fe atoms near the cluster being displaced from their original sites along the $<111>$ and $<112>$ directions, which releases the accumulated pressure. However, the maximum Fe atom displacement is less than $1 \AA$. The insertion of the $11^{\text {th }}$ $\mathrm{He}$ atom results in a second <111> SIA. Adding two more He atoms leads to a third $<111>$ SIA, and most of the displaced Fe atoms around the cluster return to their original positions. Insertion of more He atoms produces additional SIAs, but all of them are collected at the periphery of the He cluster, a similar behavior was observed in the bulk. Fig. 5 shows the annealed cluster configurations for: (a) $4 \mathrm{He}$, (b) $13 \mathrm{He}$ and (c) $25 \mathrm{He}$, where the red spheres represent He atoms, and the green and white spheres indicate $\mathrm{Fe}$ atoms. The hydrostatic stress is less than or greater than $8.0 \mathrm{GPa}$, respectively, but the stress of a crowdion is generally larger. To clearly show the configurations, the systems are quenched to 0 K. From Fig. 5, it can be seen that a $<111>$ SIA is created near the GB plane by 4 He atoms, which is a much smaller cluster than needed to form an SIA in the bulk. This may be due to the fact that the formation energy of a self-interstitial in the $\Sigma 3 \mathrm{~GB}(2.81 \mathrm{eV})$ is lower than that of $3.53 \mathrm{eV}$ in the bulk [15]. Three $<111>$ SIAs are emitted and some nearby Fe atoms are pushed off from their normal sites when the cluster reaches $13 \mathrm{He}$ atoms. Adding more He into the $\Sigma 3$ GB causes more SIAs to accumulate around the He cluster, but these SIAs no longer form $<111>$ SIAs parallel to the GB plane. Fig. 5 indicates that $<111>$ SIAs migrate 
easily parallel to the GB plane along <111> directions because of their low migration energy barrier of about $0.22 \mathrm{eV}$ in the $\Sigma 3 \mathrm{~GB}$ [7].

A dislocation loop with a Burgers vector of $\boldsymbol{b}=1 / 2<111>$ is generated when the cluster contains $45 \mathrm{He}$ atoms. Fig. 6 shows the cluster configurations before and after annealing for He clusters with 45 and $97 \mathrm{He}$ atoms. For a 45 atom cluster, before annealing, a large number of SIAs form at the cluster periphery on both sides of the GB, as shown in Fig. 6(a). Compared to the $20 \mathrm{He}$ atom cluster in Fig. 5(c), all the SIAs remain at the periphery of the cluster, except for the $<111>$ SIA in the GB plane located about $1.5 \mathrm{~nm}$ from the cluster center. During annealing most of the SIAs gather on one side of the cluster forming a $1 / 2[111]$ dislocation loop, see Fig. 6(b). Also, it can be seen that a step forms in the GB on the right hand side of the cluster. Formation of the step appears to be a consequence of transmission of a dislocation segment through the GB. Note that there are dislocation loops on both sides of the GB as shown in Fig. 6(a). The Burgers vector of the lower grain dislocation loop is $1 / 2$ [-1-11]. If this loop crosses from the lower grain to the upper grain, then a residual defect is deposited in the GB to account for the misorientation difference between the two grains. The difference between the loop dislocation Burgers vectors gives the Burgers vector of the residual dislocation remaining in the GB after the transmission occurs. The residual Burgers vector, $b_{r}$, is given by

$$
\vec{b}_{r}=\vec{b}_{l}-R \vec{b}_{u}
$$

where $R$ is the rotation matrix that maps a direction in the lower grain into the coordinate system of the upper grain, $b_{l}$ is the Burgers vector of the lower grain dislocation, and $b_{u}$ is the Burgers vector of the upper grain dislocation loop. For the $\Sigma 3$ GB examined here the rotation matrix is 


$$
R=\left[\begin{array}{ccc}
2 & -1 & 2 \\
-1 & 2 & 2 \\
-2 & -2 & 1
\end{array}\right]
$$

Inserting appropriate vectors in equation 2 gives a residual GB Burgers vector of 1/3[11-1]. Inspection of the dichromatic pattern (not shown) for the $\Sigma 3 \mathrm{~GB}$ reveals that this Burgers vector is parallel to the GB plane. Consequently, the residual GB step formed when the loop in the low grain transits to the higher grain is a disconnection, as shown by the yellow dotted line in Fig. 6(b).

With further insertion of $\mathrm{He}$ atoms, the dislocation loop is observed to grow larger by collecting more SIAs. Fig. 6(c) shows that the dislocation loop with $N_{\mathrm{I}}=42$ is still attached to the cluster or bubble with $97 \mathrm{He}$ atoms. Further annealing at $300 \mathrm{~K}$ leads to emission of the dislocation loop from the bubble, which moves away along the $<111>$ direction, as shown in Fig. 6(d).

Fig. 7 shows the configurations of the $97 \mathrm{He}-\mathrm{V}$ bubble and the associated dislocation loop after annealing for $35 \mathrm{ps.} \mathrm{During} \mathrm{annealing,} \mathrm{the} \mathrm{loop} \mathrm{is} \mathrm{observed} \mathrm{to} \mathrm{quickly} \mathrm{glide}$ along the $<111>$ direction. The loop glide cylinder and extra planes are indicated in Fig. 7 (b). Evidently the $1 / 2<111>$ loop has a $\{110\}$ habit plane, which is different from that found in bulk $\alpha$-Fe. However, the $1 / 2<111>\{110\}$ dislocation loop contains 42 SIAs in the $\sum 3$ GB that is much larger than the $1 / 2<111>\{111\}$ loop in the bulk (consisting of only 18 SIAs). The different habit planes for $<111>$ loops may be attributed to the loop size, as discussed by Osetsky et al [27] In their simulations, the position of the dislocation core for a small $1 / 2<111>$ loop with $N_{\mathrm{I}}=19$ was observed to preferentially rotate towards the pure edge (111) orientation, resulting in the (111) habit plane, but the larger $1 / 2<111>\{110\}$ loop with $N_{\mathrm{I}}>37$ did not transform into the pure edge configuration, remaining its (110) habit plane. More recently, Chen et al [43] used transmission electron microscopy, combining MD simulations, to study small interstitial dislocation loops in bcc iron, and the results demonstrated that the observed $1 / 2(111)$ loops have a wide range of different habit planes, including (110), (111) and (211). 
Considering different potentials used, the different habit planes observed in Refs. [28,35] require to be further explored.

\section{He cluster evolution in a $\Sigma 73 \mathrm{~b}$ GB}

In order to more generally understand the effects of GB structure on the growth of He bubbles and the evolution of SIAs in $\alpha-F e$, the same approach is applied to a small angle $\Sigma 73 \mathrm{~b}$ GB. The evolution of He clusters and SIAs in the $\Sigma 73 \mathrm{~b}$ GB is significantly different from that observed in the $\Sigma 3 \mathrm{~GB}$ and bulk iron. The first $\mathrm{He}$ is inserted in a most favourable interstitial site near the GB dislocation core, such that an Fe atom is pushed away from its lattice site and shares a lattice vacancy with the He atom, forming a Fe-He dumbbell along the $<111>$ direction within the GB dislocation core. When the second, third and fourth He atoms are inserted, the Fe atoms near the He atoms are displaced slightly from their original positions. After insertion of the $5^{\text {th }}$ He atom, a second Fe is emitted from the cluster. The two SIAs align along the line direction within the core of the GB dislocation, evidently being attracted by the excess volume of the core. During further insertion of He atoms, more Fe atoms around the He cluster are pushed out from their lattice sites and aggregate at the core of the GB dislocation. Almost all the He atoms distribute along the GB dislocation line, forming a longitudinal shaped cluster. Fig. 8 shows the configurations of the $\Sigma 73 \mathrm{~b}$ GB projected onto the (101) and (-616) planes, containing He clusters of two different sizes (21 and 83 atoms). For the 21 atom He cluster, He aggregates within the GB, adopting a longitudinal shape along the dislocation line. The longitudinal platelet-like shape is also observed when the number of He atoms increases to 83, a typical shape that is often observed at GBs in experiments [44]. Also, it is clear that many SIAs are formed within the dislocation core, and they prefer to remain within the core due to the large excess volume in that region, leading to strong trapping of the SIAs. Comparing Figs. 8(b) and (d), it can be seen that the GB dislocations climb in the [ $\overline{1} \overline{1} 12]$ direction. This is due to fact that 
most of the SIAs at the periphery of the He cluster prefer to align with the [ $\overline{1} \overline{1} 12]$ direction.

The results well demonstrate that the nucleation and growth of He bubbles in the bulk and GBs of bcc iron are different, and the defect behaviour or loops characteristic induced by the helium bubble growth depends on the local structure. The local structure effects on the Helium growth are also found in the experiments of He implanted copper [45]. Also, the ab initio calculations showed the nucleation and growth of the He bubbles depending on the local GB structures [11]. Here, we further explore the local structure effects of GBs by calculating the bubble pressure in the two GBs using Eq.(1), and the results are shown in Fig. 9. The pressure in the He bubbles generally decreases with increasing numbers of He atoms in the two GBs, which is the same as that in bulk Fe. The pressure drops when SIA emission or loop punching occurs in the $\Sigma 3$. It is of interest to note that the general trend of pressure change in the $\Sigma 3 \mathrm{~GB}$ is similar to that in bulk Fe, but loop punching in the $\Sigma 3$ GB leads to a larger pressure drop, which suggests that the loop punching in GBs is harder due to the stronger binding of helium bubbles to GBs [13]. Moreover, Fig. 9(b) shows that the pressure change in the $\Sigma 73 b$ GB is much different to that in the bulk and $\Sigma 3$ GB of bcc iron. The pressure sharply drops at the early stage of the helium cluster growth, but it remains almost the same after inserting the 30th He atom. At the early stage, the small He cluster in the $573 \mathrm{~b}$ GB is almost spherical, and the behaviour of the He cluster is similar to that in the bulk and the $\Sigma 3$ GB. However, as the helium bubble is getting larger, the implanted He atoms prefer to distribute along the GB dislocation line, where it has large excess volume [13], releasing its pressure without emitting SIAs or loop. This is why no loop can be detected in the $\Sigma 73$ b GB.

\section{CONCLUSION}

The nucleation and growth of He clusters or bubbles in the bulk, and at $\Sigma 3<110>$ $\{112\}$ and $\sum 73 b<110>\{661\}$ GBs in bcc iron are studied using molecular dynamics, 
and the evolution of the material structures due to He accumulation and the growth of He clusters is analysed in details. The nucleation and growth of He bubbles, as well as the formation of SIAs, at different GBs and in the bulk is significantly different, strongly correlated to microstructural features. The results show that a $1 / 2<111>\{111\}$ dislocation loop is formed with the sequential collection of $<111>$ crowdions, which are created due to the growth of He clusters, and a loop consisting of 18 SIAs is emitted from the $\mathrm{He}$ cluster when $\mathrm{He}$ atoms increase up to $41 \mathrm{He}$ atoms in the bulk. After inserting $45 \mathrm{He}$ atoms in the $\Sigma 3 \mathrm{~GB}$, a $1 / 2<111>$ dislocation loop is formed, which is attached to the He cluster. It is of interest to find that a disconnection at the GB is formed, which is a consequence of transmission of a dislocation segment through the GB. The Burgers vector of the residual dislocation remaining in the GB, as associated with the disconnection, is determined. As continuous collection of SIAs, a $1 / 2<111>\{110\}$ loop with a total of 42 SIAs is eventually emitted from the bubble with $97 \mathrm{He}$ atoms in the $\Sigma 3$ GB. The dislocation emission and GB deformation may be a main mechanism accounting for the nucleation and growth of He bubbles at large angle GBs. However, in the $\sum 73 \mathrm{~b}$ GB the insertion of He atoms induces the GB dislocation to migrate along the $[\overline{1} \overline{1} 12]$ direction, which is attributed to the aggregation and reconstruction of the SIAs at the core of the GB dislocation. Also, it is observed that the He bubbles form spherical shapes in the bulk and $\Sigma 3 \mathrm{~GB}$, but in the $\Sigma 73 \mathrm{~b}$ GB they are a longitudinal shape along the dislocation line.

\section{ACKNOWLEDGEMENTS}

L.Yang and X.T. Zu are grateful for the support by the National Natural Science Foundation of China — NSAF (Grant No: U1430109). S.M. Peng and X.G. Long and X.S. Zhou are grateful for the Science and Technology Foundation of China Academy of Engineering Physics (Grant No: 2010A0301011). F. Gao, and R.J. Kurtz are grateful for support by the US Department of Energy/Office of Fusion Energy Sciences under Contract DE-AC06-76RLO 1830. 


\section{REFERENCES}

[1] M.J. Demkowicz, R.G. Hoagland, J.P. Hirth, Phys. Rev. Lett. 100 (2008) 136102136105.

[2] G.J. Ackland, Science 26 (2010) 1587-1588.

[3] L.L. Liu, Z. Tang, W. Xiao, Z. Wang, Mater. Lett. 109 (2013) 221-224.

[4] X.M. Bai, A.F. Voter, R.G. Hoagland, M. Nastas, B.P. Uberuaga, Science 327 (2010) 1631-1634.

[5] D. Terentyev, X. He, Comput. Mater. Sci. 50 (2011) 925-933.

[6] L. Yang, X.T. Zu, H.Y. Xiao, F. Gao, H.L. Heinisch, R.J. Kurtz, K.Z. Liu, Appl. Phys. Lett. 88 (2006) 091915-91917.

[7] X. T. Zu, L. Yang, F. Gao, S. M. Peng, H. L. Heinisch, X. G. Long, R. J. Kurtz. Phys. Rev. B 80 (2009) 054104-54109.

[8] F. Sefta, K.D. Hammond, N.Juslin, B.D. Wirth, Nucl. Fusion 53 (2013) 07301573021.

[9] K.O.E. Henriksson, K. Nordlund, J. Keinonen, Nucl. Instr. Meth. B 244 (2006) 377391.

[10] J Wang, Q. Hou, T. Sun, XG. Long, X.C. Wu, S.Z. Luo, J Appl. Phys. 102 (2007) 093510-93513.

[11] L. Zhang, C.C. Fu. G.H. Lu, Phys. Rev. B 87 (2013) 134107-134117.

[12] H.Q. Deng, W.Y. Hu, F. Gao, H.L. Heinisch, S.Y. Hu, Y.L. Li, R.J. Kurtz, J. Nucl. Mater. 442 (2013) S667-S673.

[13] L. Yang, F. Gao, H.L. Heinisch, R.J. Kurtz, D. Terentyev, X.T. Zu, Acta Mater. 82 (2015) 275-286.

[14] L. Yang, H.Q. Deng, F. Gao, H.L. Heinisch, R.J. Kurtz, S.Y. Hu, Y.L. Li, X.T. Zu, Nucl. Instr. Meth. B 303 (2013) 68-71.

[15] M.A. Tschopp, F. Gao, L. Yang, K.N. Solanki. J. Appl. Phys. 115 (2014) 03350333518.

[16] M. A. Tschopp, F. Gao, K. N. Solanki. J, Appl. Phys. 115 (2014) 233501-233515.

[17] G.J. Ackland, M.I. Mendelev, D.J. Srlolovitz, S. Han, A.V. Barashev, J. Phys.: 
Condens. Matter 16 (2004) S2629-S2642.

[18] F. Gao, H.Q. Deng, H.L. Heinisch, R.J. Kurtz, J. Nucl. Mater. 418 (2011) 115-120.

[19] R.A. Aziz, A.R. Janzen, M.R. Moldover, Phys. Rev. Lett. 74 (1995) 1586-1589.

[20] N. Gao, H.Van Swygenhoven, M. Victorian, J. Chen, J. Phys.: Condens. Matter 23 (2011) 442201-442205.

[21] L. Yang, X.T. Zu, Z.G. Wang, H.T. Yang, F, Gao, H.L. Heinisch, R.J. Kurtz, J. Appl. Phys. 103 (2008) 063528-63536.

[22] K. Morishita, R. Sugano, B.D. Wirth, J. Nucl. Mater. 323 (2003) 243-250.

[23] S.H. Guo, B.E. Zhu, W.C. Liu, Z.Y. Pan, Y.X. Wang, Nucl. Instr. Meth. B 267 (2009) 3278-3281.

[24] D. Stewart, Y.N. Osetsky, R. Stoller, J. Nucl. Mater. 417 (2011) 1110-1114.

[25] W.D. Wilson, C.L. Bisson, M.I. Baskes, Phys. Rev. B 24 (1981) 5616-5624.

[26] A.E. Ward, S.B. Fisher, J. Nucl. Mater. 166 (1989) 227.

[27] Y.N. Osetsky, A. Serra, B.N. Singh, S.I. Golubov, Philo. Mag. A 80 (2000) 21312157.

[28] D.B. Mizandronsev, A.A. Vasiliev, In Third International Workshop on Nondestructive Testing and Computer Simulations in Science and Engineering, I.M. Alexander (Ed.), Proceedings of SPIE 4064 (2000) 220

[29] J. Marian, B.D. Wirth, .M. Perlado, Phy. Rev. Lett. 88 (2002) 255507-255510.

[30] F. Willaime, C.C. Fu, M.C. Marinica, J.D. Torre, Nucl. Inst. Meth. B 228 (2005) 92-99.

[31] B. Yao, D.J. Edwards, R.J. Kurtz, J. Nucl. Mater. 434 (2013) 402-410.

[32] J. Chen, P. Jung, W. Hoffelner, and H. Ullmaier, Acta Mater. 56 (2008) 250-258.

[33] S.L. Dudarev, R. Bullough, P.M. Derlet, Phy. Rev. Lett. 100 (2008) 135503135506.

[34] H.X. Hu, R.E. Stoller, Y.N. Osetsky, D. Terentyev, Phy. Rev. Lett. 110 (2013) 265503-265507.

[35] E. Kuramoto, K. Ohsawa, J. Imai, K. Obata, T. Tsutsumi, J. Nucl. Mater. 329-333 
(2004) 1223-1227.

[36] L. Yang, Z.Q. Zu, S.M. Peng, X.G. Long, X.S. Zhou, X.T. Zu, H.L. Heinisch, R.J. Kurtz, F. Gao, J. Nucl. Mater. 441 (2013) 6-14.

[37] C.H. Rycroft, G.S. Grest, J.W. Landry, M. Z. Bazant. Phys. Rev. E 74 (2006) 021306-021321.

[38] P.L. Lane, P.J. Goodhew, Philo. Mag. A 48 (1983) 965.

[39] V. Randle, Acta Metall. Mater. 39 (1991) 481-492.

[40] K. Ono, M. Miyamoto, K. Arakawa, J. Nucl. Mater. 367-370 (2007) 522-526.

[41] R.J. Kurtz, H.L. Heinisch, J. Nucl. Mater. 329 (2004)1199-1203.

[42] F. Gao, H.L. Heinisch, R.J. Kurtz, J. Nucl. Mater. 386-388 (2009) 390-394.

[43] J. Chen, N. Gao, P. Jung, J. Nucl. Mater. 441 (2013) 216-221.

[44] H. Ullmaier (Ed.), Fundamental Aspects of Helium in Metals (Proc. Int. Symp. Julich, 1982), Radiat. Eff. 78 (1983) 1.

[45] P.A. Thorsen, J.B. Bilde-Sorensen, B.N. Singh, Scripta Mater. 51 (2004) 557-560. 

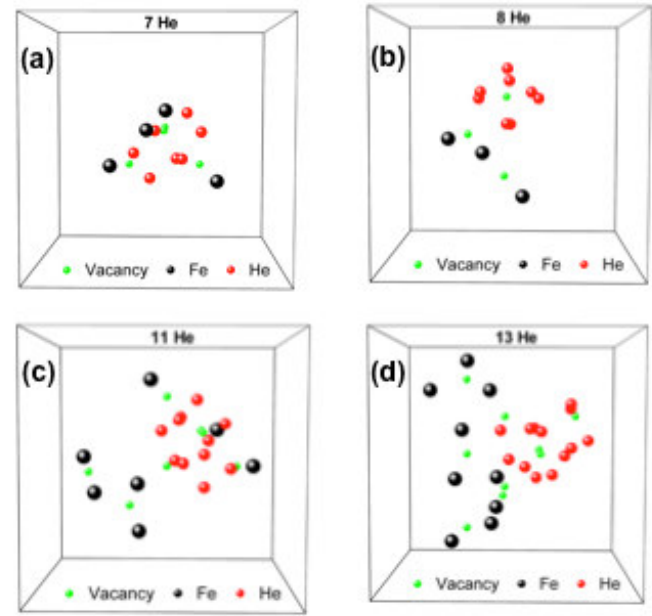

Fig. 1. Atomic configurations of the He cluster and its nearby displaced Fe atoms after adding He atoms followed annealing $0.1 \mathrm{~ns}$ at $300 \mathrm{~K}$ : (a) $7 \mathrm{He}$, (b) $8 \mathrm{He}$, (c) 11 He and (d) $13 \mathrm{He}$, where large black, medium red and small green spheres present SIAs, He and vacancies, respectively. 

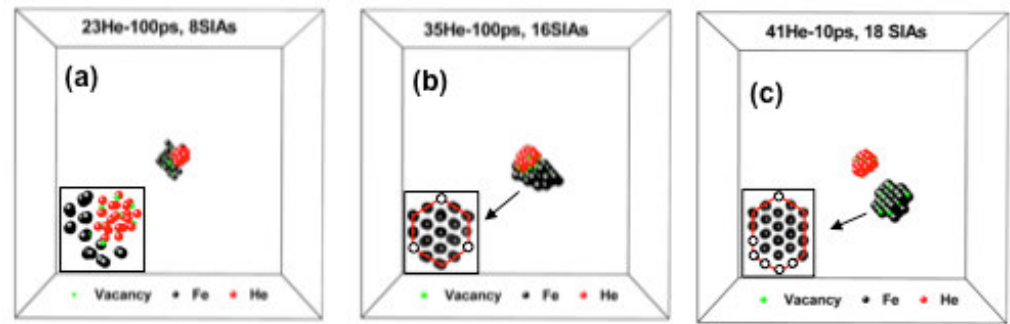

Fig. 2. Annealed configurations of the defects and He atoms at $300 \mathrm{~K}$ after inserting: (a)

$23 \mathrm{He}$, (b) $35 \mathrm{He}$, (c) $41 \mathrm{He}$, where the representation of spheres are the same as those in

Fig. 1. 
(a)

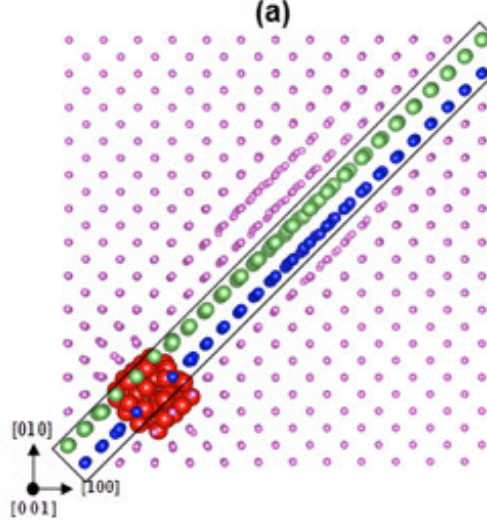

(b)

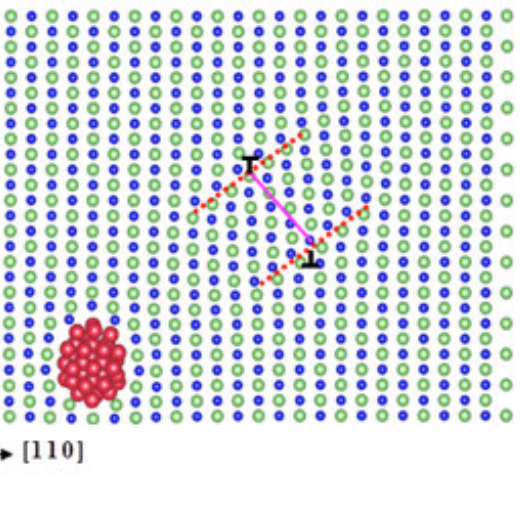

Fig. 3. Configurations of the 41-He cluster and its nearby Fe atoms after annealing $10 \mathrm{ps}$ in the bulk of Fe: (a) projected on the (001) plane, (b) two Fe atomic layers in the rectangle of (a) projected on the $(-110)$ plane together with the He clusters, where the large red spheres are He atoms, and the other spheres indicate Fe atoms. 


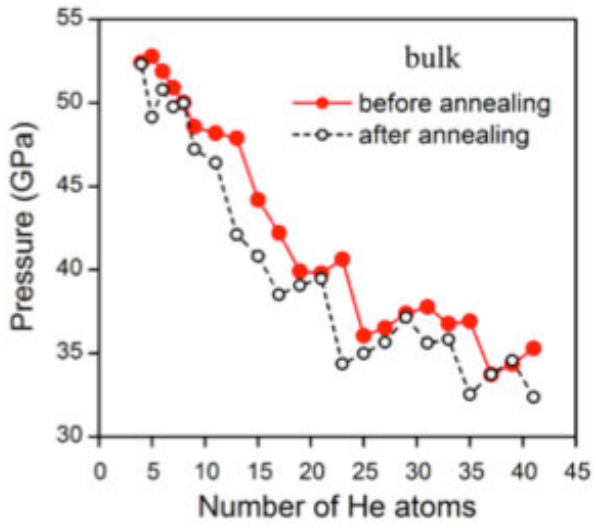

Fig. 4. Pressure of He clusters before and after annealing in the bulk of bcc iron. 

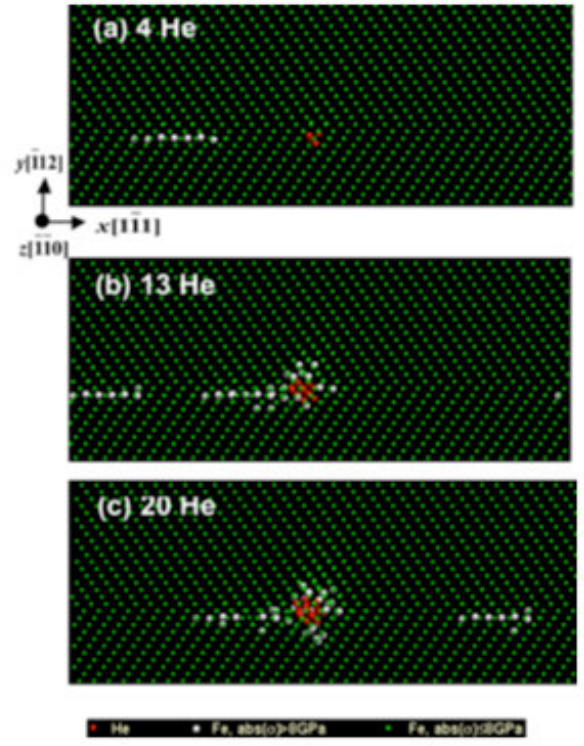

Fig. 5. Atomic configuration of the $\Sigma 3 \mathrm{~GB}$ and He clusters: (a) $4 \mathrm{He}$, (b) $13 \mathrm{He}$ and (c) $20 \mathrm{He}$, where red spheres are He atoms, white spheres represent the Fe atoms with the stress of $8 \mathrm{GPa}$ and larger, green spheres represent the Fe atoms with the stress of less than $8 \mathrm{GPa}$. 


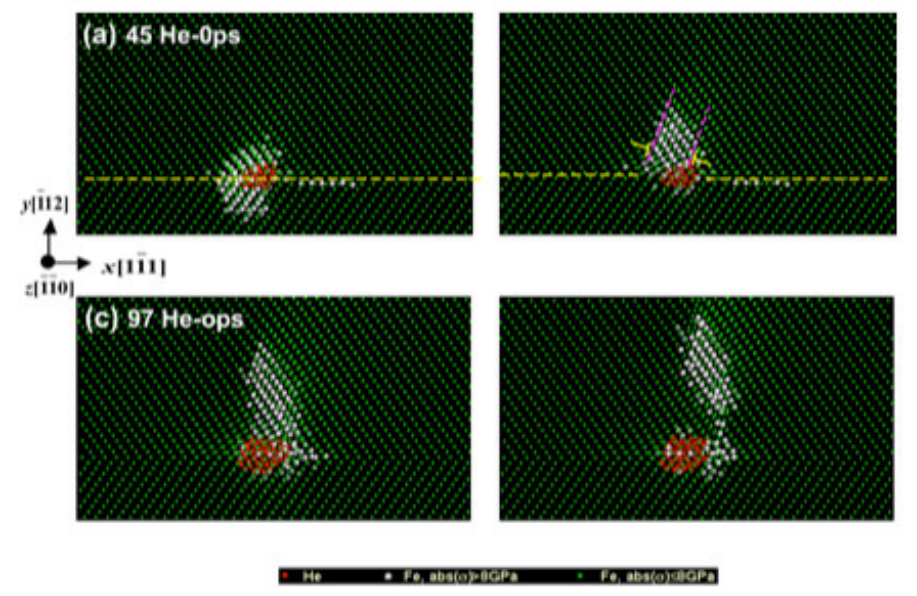

Fig. 6. Atomic configurations of the $\Sigma 3$ GB together with the He clusters in $\alpha$-Fe: (a) before and (b) after annealing $0.1 \mathrm{~ns}$ at $300 \mathrm{~K}$ for $45 \mathrm{He}$ atoms, (c) before and (d) after annealing $35 \mathrm{ps}$ at $300 \mathrm{~K}$ for $97 \mathrm{He}$ atoms, where the representation of the spheres are the same as those in Fig. 5. Yellow dashed lines indicate the grain boundary plane. 


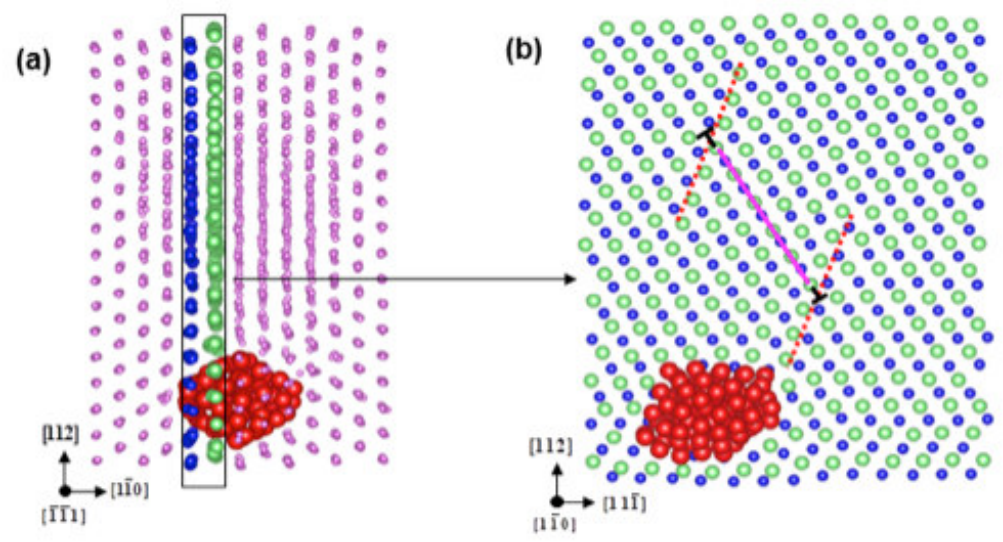

Fig. 7. Atomic configurations of the $97-\mathrm{He}$ cluster and its nearby Fe atoms after annealing 35 ps: (a) projected on the (-11-1) plane, (b) two Fe atomic layers in the rectangle of (a) projected on the $(-1-10)$ plane together with the He clusters, where the large red spheres are He atoms, and the other spheres indicate Fe atoms. 


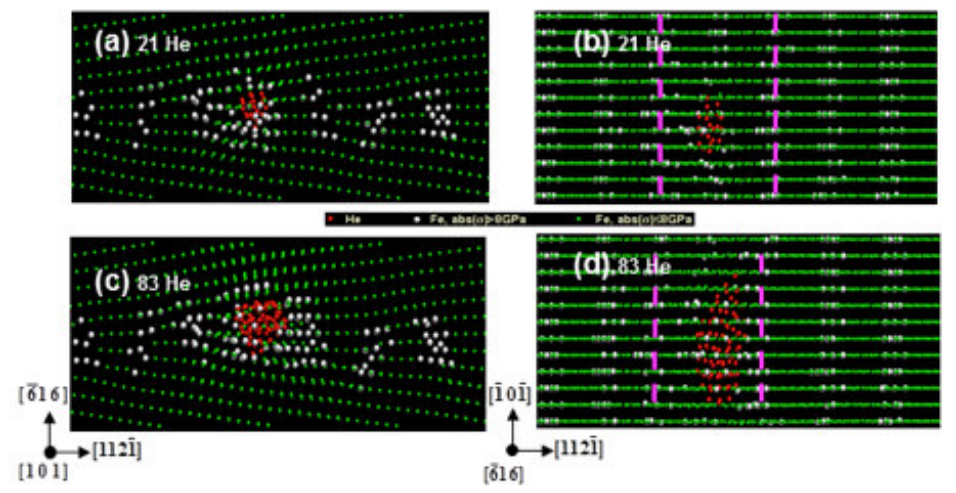

Fig. 8. Atomic configurations of the $\Sigma 73 \mathrm{~b}$ GB together with the cluster of $21 \mathrm{He}$ atoms projected on: (a) the (101) plane and (b) the (-616) plane, as well as the cluster of $83 \mathrm{He}$ atoms projected on: (c) the (101) plane and (d) the $(-616)$ plane, where the representation of spheres are the same as those in Fig. 5. 

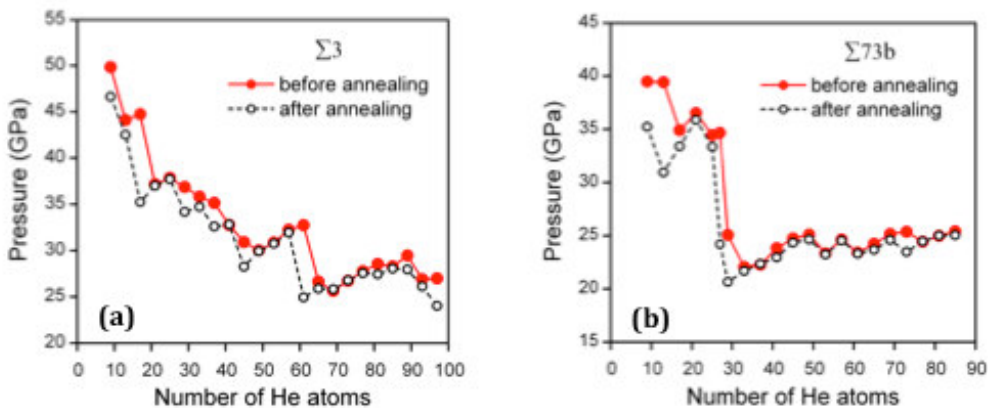

Fig. 9. Pressure of He clusters before and after annealing in the GBs of bcc iron: (a) $\Sigma 3$ and (b) $\Sigma 73 b$. 


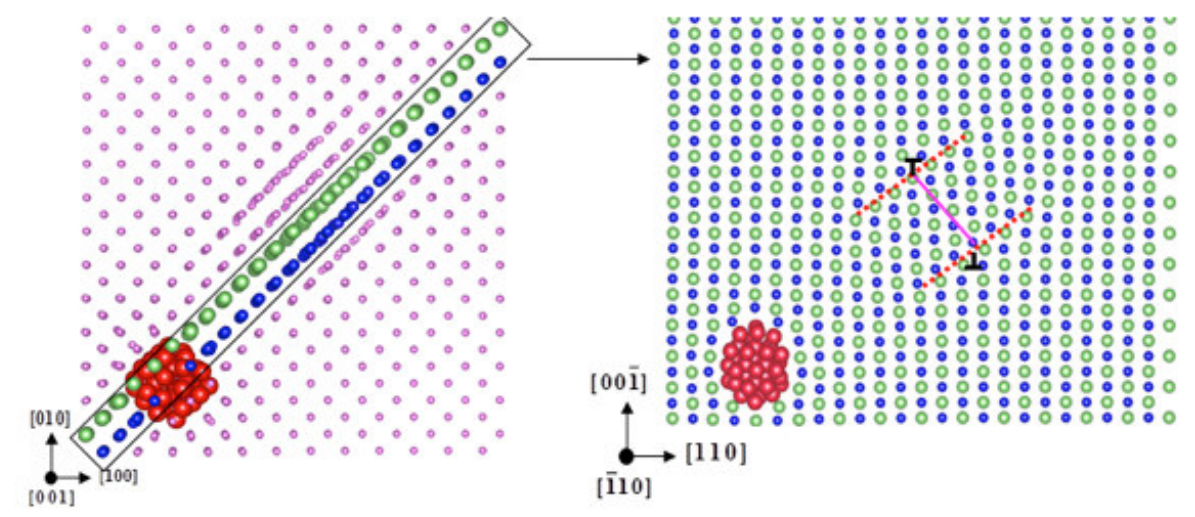

Graphical abstract 